\title{
Innovación en la capacitación docente online: un modelo organizacional para brindar apoyo a largo plazo a la docencia online
}

\section{Innovations in online faculty development: an organizational model for long-term support of online faculty}

\author{
Dr. Laurence B. BOGGESS. Director. The Pennsylvania State University (Ibb150@psu.edu).
}

\section{Resumen:}

En 2008, la organización de enseñanza online de la Pennsylvania State University, el Penn State World Campus, creó una unidad dedicada a la capacitación docente para mejorar la formación online. La unidad de capacitación docente online (en adelante OFD, del inglés Online Faculty Development) fue diseñada con el objetivo de preparar al profesorado para la enseñanza online a través de un programa amplio y variado de cursos asíncronos de formación online basados en la teoría e investigación de las mejores prácticas relacionadas con la instrucción online. En este documento se describe la unidad OFD del Penn State World Campus, su misión y programa, y las premisas sobre las que se basan sus operaciones diarias.
Descriptores: capacitación docente online, enseñanza online, desarrollo profesional, competencias de enseñanza online, formación asíncrona, certificado de enseñanza online.

\section{Abstract:}

In 2008, Penn State University's online delivery organization, Penn State World Campus, established a dedicated faculty development unit to improve online instruction. The Online Faculty Development (OFD) unit was designed to prepare faculty for online teaching through a large and varied curriculum of asynchronous, online training courses built on the theory and research of best practices for online instruction. This paper describes the Penn State World Campus OFD unit, its mission

Fecha de recepción de la versión definitiva de este artículo: 18-07-2019.

Cómo citar este artículo: Boggess, L. B. (2020). Innovación en la capacitación docente online: un modelo organizacional para brindar apoyo a largo plazo a la docencia online | Innovations in online faculty development: an organizational model for longterm support of online faculty. Revista Española de Pedagogía, 78 (275), 73-87. doi: https://doi.org/10.22550/REP78-1-2020-01 
and program, and the assumptions underlying its day-to-day operations.
Keywords: online faculty development, online teaching, professional development, online teaching competencies, asynchronous training, online teaching certificate.

\section{Introducción}

La educación online se ha convertido en un sistema habitual de impartición de programas académicos de grado y cursos universitarios. Sin embargo, la enseñanza online puede ser una experiencia muy distinta a la presencial debido a que el entorno de aprendizaje (asíncrono, a distancia y digital) todavía es nuevo para muchos profesores que provienen del formato presencial. La impartición de enseñanza online requiere formación especializada diseñada para desarrollar las mejores prácticas, los conocimientos, las habilidades y las comde los alumnos. Las razones que justifican la afirmación anterior son:

1) Pocas habilidades y competencias de la enseñanza presencial se transfieren a la enseñanza online.

2) Muchos profesores con experiencia, pero nuevos en la enseñanza online, desconocen de primera mano la metodología de aprendizaje de los estudiantes online.

3) Para diseñar e impartir cursos online es necesario comprender la teoría del aprendizaje, por ejemplo, saber alinear los resultados de aprendizaje de los alumnos, las evaluaciones y las tareas, diseñar temas de debate efectivos 0 crear proyectos de grupo online eficaces.

Para muchos instructores, tanto presenciales como online, la capacitación docente online constituye su primera introducción formal a la enseñanza. Según la iniciativa de aprendizaje Educause, asociación líder en formación universitaria de Estados Unidos, el principal problema de la enseñanza y el aprendizaje en 2017 y 2019 fue la capacitación docente (Educause, 2017, 2019). Dado que el aprendizaje online ha llegado para quedarse, las universidades deberían centrar su atención en formar al profesorado para que sea capaz de impartir enseñanza online con un nivel alto de competencia y calidad. En este documento se describe la teoría y la práctica en las que se basa una universidad al afirmar que los cursos online deben ser impartidos por instructores formados específicamente a fin de mejorar y mantener la calidad, garantizar el aumento de las matrículas y el éxito de los estudiantes, y consolidar la marca institucional a través de una formación de calidad.

La capacitación docente online (OFD) es un subconjunto relativamente nuevo de la capacitación docente universitaria que cuenta con un cuerpo de investigación 
cada vez mayor, lo que respalda su importancia en la educación online y fomenta las mejores prácticas (por ejemplo, Allen y Seaman, 2007; Bigatel, Ragan, Kennan, May y Redmond, 2012; Gregory y Martindale, 2016; McQuiggan, 2012; Palloff y Pratt, 2011). La OFD acepta premisas básicas relativas a la eficacia de la enseñanza y el aprendizaje online:

1) Aunque "la buena enseñanza, buena enseñanza es", se necesitan habilidades, conocimientos y competencias específicos para impartir formación asíncrona online que no son intuitivos ni autodidácticos para un profesor presencial que se estrena en este tipo de enseñanza.

2) Pocas técnicas de enseñanza presencial pueden trasladarse al espacio online, mientras que muchas prácticas online influyen positivamente y mejoran la enseñanza presencial.

3) Las investigaciones se están centrando en las mejores prácticas a fin de fomentar una instrucción online eficaz y la participación de los alumnos.

4) Un compromiso institucional con la OFD, incluido personal experto y dedicado, financiación constante y un plan a largo plazo a nivel de universidad, son fundamentales para preparar a los profesores para el presente y el futuro del aprendizaje universitario online.

Además, como práctica de desarrollo profesional, la OFD está impulsada por la innovación y es sensible a esta. En la enseñanza presencial de las universidades estadounidenses, rara vez los profesores adjuntos recién contratados requieren capacitación docente. En dicho formato, siempre se ha dado por sentado que los expertos en la materia también son expertos en la enseñanza, una suposición falsa que puede dar lugar a una instrucción ineficaz. En cambio, la movilización de las universidades a la hora de requerir algún tipo de preparación formal para la enseñanza online constituye una gran innovación en la educación superior estadounidense. La capacitación previa a la impartición de enseñanza online está motivada por varios factores:

1) La aceptación de que el espacio de aprendizaje digital asíncrono se diferencia lo bastante del formato presencial como para que sea necesaria formación especializada.

2) La asignación de recursos a disposición del "enfoque de equipo" consistente en trasladar los cursos y programas presenciales al espacio online.

3) La promesa de nuevos ingresos derivados de las matrículas de la formación online.

4) La posibilidad de conseguir una mejor posición en las clasificaciones universitarias mediante una extensa formación en la enseñanza online.

A través de la normalización de la formación y la acreditación de los profesores online vemos una tendencia que confirma la relación entre desarrollo docente profesional, instrucción de calidad y éxito de los estudiantes. 


\section{Mejores prácticas para la for- mación online}

Chickering y Gamson (1987) identificaron siete principios para lograr el éxito de la enseñanza universitaria. Estos se expresan como comportamientos del profesor, identificables como competencias y conocimientos:

1. Fomentar el contacto entre alumnos y profesores.

2. Desarrollar reciprocidad y cooperación entre los alumnos.

3. Fomentar el aprendizaje activo.

4. Proporcionar retroalimentación rápida.

5. Subrayar el tiempo destinado a una tarea.

6. Comunicar las altas expectativas.

7. Respetar los diversos talentos y formas de aprendizaje.

Estos siete comportamientos no son mera teoría; cada uno de ellos puede aplicarse en la planificación, la formación guiada, la práctica y la fijación de objetivos y así influir positivamente en el aprendizaje y éxito de los alumnos. En la base de estos comportamientos hay varios factores clave:

1) La importancia de establecer relaciones a través de la enseñanza y el aprendizaje.

2) La posición centrada en el alumno del profesor.
3) El valor de la contribución del alumno en el diseño y la gestión del curso.

4) La necesidad de una buena alineación entre los componentes básicos de un curso: objetivos de aprendizaje del alumno, tareas, evaluaciones y diseño del curso.

5) La importancia de la relación del alumno con el profesor, sus compañeros y el contenido y programa del curso.

En la formación universitaria online, estos comportamientos siguen siendo relevantes, pero deben conceptualizarse y ajustarse para adaptarlos a un espacio demográfico y de aprendizaje diferente: el estudiante adulto online y el alumno en edad universitaria convencional que aprenden de forma asíncrona, en distintas zonas horarias y a través de una interfaz digital. Trasladados al entorno online, los comportamientos docentes descritos por Chickering y Gamson (1987) deben aprovechar las posibilidades del aprendizaje online e innovar en torno a las limitaciones inherentes al espacio digital asíncrono. Por ejemplo, los comportamientos "fomentar el contacto entre alumnos y profesores" y "respetar los diversos talentos y formas de aprendizaje" pueden ser más productivos online porque, a diferencia de lo que sucede en las conferencias o debates presenciales, en los foros de discusión se escucha la opinión de todos los alumnos. Las cuestiones de raza, etnia, clase 0 accesibilidad física desaparecen online, lo que permite una mayor igualdad de las contribuciones y el intercambio de diversos puntos de vista y experiencias vitales. 
Una investigación basada en las conclusiones de Chickering y Gamson determinó que estas mejores prácticas de la educación presencial podrían traducirse en mejores prácticas online y generar múltiples habilidades y conocimientos específicos relevantes para el aprendizaje asíncrono. Bigatel et al. (2012) señalaron tres catego- rías de competencias docentes online que abarcan la enseñanza online: competencias pedagógicas, tecnológicas y administrativas (véase la Tabla 1). Se determinó que cada una de estas mejores prácticas guarda una relación positiva con la participación del alumno, un indicador fiable del aprendizaje y éxito de los estudiantes.

TABLA 1. Competencias docentes online.

\begin{tabular}{|c|c|c|}
\hline Pedagógicas & Técnicas & Administrativas \\
\hline $\begin{array}{l}\text { Prestar atención a los singula- } \\
\text { res desafíos del aprendizaje a } \\
\text { distancia, donde los alumnos } \\
\text { se encuentran separados por } \\
\text { el tiempo y la proximidad } \\
\text { geográfica y las interacciones } \\
\text { son principalmente de carácter } \\
\text { asíncrono. }\end{array}$ & $\begin{array}{l}\text { Realizar operaciones } \\
\text { informáticas básicas. }\end{array}$ & $\begin{array}{l}\text { Registrarse en el curso y parti- } \\
\text { cipar activamente. }\end{array}$ \\
\hline $\begin{array}{l}\text { Familiarizarse con las necesi- } \\
\text { dades y situaciones de apren- } \\
\text { dizaje particulares tanto de los } \\
\text { alumnos adultos como de los } \\
\text { alumnos en edad universitaria } \\
\text { convencional, proporcionando } \\
\text { una experiencia educativa } \\
\text { que sea apropiada para ambos } \\
\text { tipos. }\end{array}$ & $\begin{array}{l}\text { Registrarse correcta- } \\
\text { mente en el sistema de } \\
\text { gestión del aprendizaje } \\
\text { y acceder al curso. }\end{array}$ & $\begin{array}{l}\text { Comunicar a los alumnos } \\
\text { cuándo se calificarán y devol- } \\
\text { verán las tareas y los exáme- } \\
\text { nes. }\end{array}$ \\
\hline $\begin{array}{l}\text { Dominar el contenido, la } \\
\text { estructura y la organización } \\
\text { del curso. }\end{array}$ & $\begin{array}{l}\text { Navegar correctamente } \\
\text { por el entorno del curso. }\end{array}$ & $\begin{array}{l}\text { Ofrecer un plan de estudios } \\
\text { completo que se adhiera a la } \\
\text { política institucional de plan } \\
\text { de estudios. }\end{array}$ \\
\hline $\begin{array}{l}\text { Responder a las consultas de } \\
\text { los alumnos. }\end{array}$ & $\begin{array}{l}\text { Configurar y gestionar } \\
\text { las calificaciones de los } \\
\text { alumnos. }\end{array}$ & $\begin{array}{l}\text { Mediar en conflictos de los } \\
\text { alumnos relacionados con el } \\
\text { curso. }\end{array}$ \\
\hline $\begin{array}{l}\text { Proporcionar retroalimenta- } \\
\text { ción detallada sobre tareas y } \\
\text { exámenes. }\end{array}$ & $\begin{array}{l}\text { Utilizar de manera } \\
\text { efectiva los sistemas de } \\
\text { comunicación del curso. }\end{array}$ & $\begin{array}{l}\text { Cumplir las políticas ins- } \\
\text { titucionales relativas a los } \\
\text { derechos de los alumnos y a la } \\
\text { privacidad y accesibilidad. }\end{array}$ \\
\hline $\begin{array}{l}\text { Comunicarse con los alumnos } \\
\text { acerca del progreso y los cam- } \\
\text { bios del curso. }\end{array}$ & $\begin{array}{l}\text { Gestionar la lista de } \\
\text { asistencia del curso. }\end{array}$ & $\begin{array}{l}\text { Revisar el contenido del curso } \\
\text { y los materiales académicos en } \\
\text { función de la retroalimenta- } \\
\text { ción de los alumnos. }\end{array}$ \\
\hline $\begin{array}{l}\text { Promover y fomentar un am- } \\
\text { biente de aprendizaje seguro, } \\
\text { acogedor y respetuoso. }\end{array}$ & $\begin{array}{l}\text { Gestionar los envíos de } \\
\text { los alumnos. }\end{array}$ & $\begin{array}{l}\text { Saber dónde y cuándo pueden } \\
\text { obtener usted y sus alumnos } \\
\text { asistencia técnica y soporte. }\end{array}$ \\
\hline
\end{tabular}




\begin{tabular}{|l|l|l|}
\hline $\begin{array}{l}\text { Supervisar y gestionar el pro- } \\
\text { greso de los alumnos. }\end{array}$ & $\begin{array}{l}\text { Gestionar los archivos y } \\
\text { carpetas del curso con- } \\
\text { tenidos en el sistema de } \\
\text { gestión del aprendizaje } \\
\text { (cuando proceda). }\end{array}$ & $\begin{array}{l}\text { Comunicar las expectativas } \\
\text { relativas al comportamiento } \\
\text { de los alumnos en el curso. }\end{array}$ \\
\hline $\begin{array}{l}\text { Comunicar los objetivos y } \\
\text { resultados del curso. }\end{array}$ & $\begin{array}{l}\text { Conocer las políticas institu- } \\
\text { cionales relativas a integridad } \\
\text { académica institucional, infor- } \\
\text { mar de estas a los alumnos y } \\
\text { supervisar su cumplimiento. }\end{array}$ \\
\hline $\begin{array}{l}\text { Aportar pruebas a los alumnos } \\
\text { de su presencia en el curso de } \\
\text { manera regular. }\end{array}$ & $\begin{array}{l}\text { Informar de las calificaciones a } \\
\text { los alumnos y registrar estas en } \\
\text { el sistema de calificaciones de la } \\
\text { universidad, según se requiera. }\end{array}$ \\
\hline $\begin{array}{l}\text { Demostrar sensibilidad ante } \\
\text { discapacidades y diversidades, } \\
\text { incluidas diferencias culturales, } \\
\text { cognitivas, emocionales y físicas. }\end{array}$ & & \\
\hline
\end{tabular}

Fuente: The Pennsylvania State University, 2019.

Diehl (2016) incluye además el diseño educativo como competencia relacionada con la enseñanza y actualiza también la investigación sobre la competencia docente online y la evolución normativa en relación con la formación online para Quality Matters, un estándar basado en categorías para el diseño de cursos online.

Aunque estas mejores prácticas guardan relación con una formación presencial eficaz, los factores de distancia, asincronía, tecnología de evolución rápida y datos demográficos de los alumnos online sugieren la necesidad de una capacitación especializada para los profesores online. Para tal fin, la Pennsylvania State University creó una unidad dedicada de capacitación docente en la estructura organizativa del World Campus cuyo objetivo era brindar apoyo a todo el cuerpo docente de dicha universidad que impartiera enseñanza online. Este apoyo inicial tuvo como resultado el desarrollo de un programa de cursos de formación online que se puso a disposición del profesorado de forma gratuita. A los términos "capacitación docente" se le añadió el descriptor "online" para distinguir las experiencias de aprendizaje específicas necesarias para adquirir las habilidades, los conocimientos y las competencias requeridos para impartir una formación online eficaz.

\section{Una unidad dedicada para la capacitación docente online en la Pennsylvania State University}

La unidad de Capacitación docente online (OFD) del Penn State World Campus se creó en 2008 con el objetivo de ofrecer cursos asíncronos online diseñados para ayudar al profesorado de la Pennsylvania State University a adquirir los conocimientos y las competencias necesarios en la enseñanza online. La OFD forma parte de la Organización de Asuntos Académicos del World Campus, junto con las unidades de diseño de aprendizaje, planificación y ges- 
tión de programas, asesoramiento académico, asuntos estudiantiles e información y tecnología de divulgación. Fundada en los inicios del World Campus y aprobada por la administración del Penn State Outreach como recurso oficial de apoyo para una formación online de calidad, la unidad OFD se financia a través del reparto de beneficios derivados de las matrículas entre el World Campus (el proveedor de la plataforma) y el programa académico y su facultad (el proveedor del contenido y la formación).

Una de las singulares características del diseño de la iniciativa online de la Pennsylvania State University es la división de responsabilidades entre el World Campus y las facultades de la universidad. El World Campus proporciona a las facultades la plataforma online y el soporte técnico para que estas ofrezcan sus programas académicos, pero no tiene ninguna autoridad sobre el contenido académico, la contratación, la evaluación del desempeño del profesorado 0 las tareas del curso. El World Campus puede asesorar a los programas académicos que deseen crear grados online y puede ayudar a comercializar dichos programas, pero solo desempeña un papel de apoyo en la calidad académica. Puede considerarse que la relación entre el Penn State World Campus y las facultades se corresponde con un diseño descentralizado, donde las facultades conservan su autoridad académica. Todos los cursos del World Campus son diseñados e impartidos por el cuerpo docente de la Pennsylvania State University. Este diseño significa que en todos los títulos obtenidos online a través del World Campus pone "Penn State" (Pennsylvania State University), no "Penn State World Campus".
Gracias a esta estructura organizativa, la unidad OFD del World Campus ha disfrutado de un apoyo y libertad que le han permitido convertirse en un líder innovador basado en la investigación cuyo objetivo es mejorar la enseñanza online en toda la universidad, así como contribuir al ámbito especializado del desarrollo profesional docente universitario. Todos los cursos de formación online basados en la investigación, los servicios personalizados y el asesoramiento al profesorado de la unidad se ofrecen de forma gratuita a los programas y profesores individuales.

La unidad OFD del Penn State World Campus ofrece a todos los profesores y alumnos de postgrado de la Pennsylvania State University un programa de casi 20 cursos de capacitación docente (denominados "cursos OL"). La mitad del programa consiste en cursos autodirigidos cuya duración total es de tres a cinco horas. El resto son cursos dirigidos por un instructor que pueden completarse en cuatro o cinco semanas y han sido diseñados para imitar un curso académico online con tareas sujetas a calificación y fechas límite que giran en torno a temas relacionados con las mejores prácticas de la enseñanza online. Los cursos requieren entre tres y cinco horas semanales y son impartidos por profesores de la Pennsylvania State University que han obtenido el Foundations of Online Teaching Certificate (Certificado de Fundamentos de la Enseñanza Online) de la unidad OFD del World Campus.

La teoría de la acción que respalda el diseño de los cursos OL se basa en la idea de que la mejor forma que existe para que un instructor aprenda a impartir formación on- 
line es adquirir conocimientos y competencias a través del aprendizaje online. En un curso OL, el profesor, especialmente si se estrena en la educación online, se convierte en alumno y va familiarizándose con el sistema de gestión del aprendizaje, experimentando las ventajas del aprendizaje social que aportan los debates asincrónicos, comprendiendo el punto de vista de los alumnos online $\mathrm{y}$ adquiriendo conocimientos y experiencia en el ámbito de la enseñanza. Además del aprendizaje de contenidos, las evaluaciones de los cursos OL han demostrado de manera consistente que aquellos profesores que comienzan un curso con una actitud reacia, temerosa 0 insegura hacia la enseñanza online terminan afirmando haber adquirido mayor confianza y competencia, factores clave en la autoeficacia de la enseñanza online (Horvitz, Beach, Anderson y Xia, 2015). del World Campus y la Pennsylvania State University comprendieron que, para crear y desarrollar una iniciativa online, era necesario asegurarse de que el profesorado recibiera formación guiada y formal que les permitiera impartir clases en los nuevos espacios de aprendizaje digital. La unidad OFD se incorporó en la estructura organizativa del World Campus, en lugar de hacerlo en la de alguna facultad o la oficina del rector, ya que fue designada como el principal apoyo del profesorado que impartía los cursos ofrecidos a través del World Campus. Financiada a través del reparto de beneficios derivados de las matrículas entre el World Campus y las facultades, la OFD fue considerada uno de los servicios prestados junto con el de asistencia al alumno, diseño de aprendizaje, asesoramiento académico universitario, soporte informático y marketing. En los últimos años, los servicios se han ampliado para incluir asuntos estudiantiles online.

Todo el personal docente que imparte clases online en la Pennsylvania State University, ya sea a través de programas de grado del World Campus o de cursos desarrollados en plataformas web a nivel de facultad, recibe formación docente online. Aunque todo el profesorado requiere algún tipo de capacitación, las fuentes de esta varían. La formación se puede recibir a nivel de programa académico, a través de la oficina de capacitación docente de la facultad o por medio de la unidad OFD del World Campus. La tendencia actual en los últimos años ha sido que las facultades y los programas académicos exigieran a sus profesores online asistir a cursos OL de la OFD.

El World Campus ofrece completamente online más de 160 programas de grado y certificación a 20000 estudiantes. Todos los semestres, aproximadamente 1200 profesores de la Pennsylvania State University imparten cursos a través del World Campus. La misión de la OFD es proporcionar a todos los profesores que imparten clases a través del World Campus preparación inicial y desarrollo docente continuo. A pesar de este gran alcance, la OFD está diseñada de forma austera. La unidad consta de cuatro miembros con responsabilidades específicas: un director que es profesor universitario y se encarga de supervisar el plan de estudios y a los instructores de los cursos OL, un subdirector que tiene un doctorado y experiencia en el diseño de apren- 
dizaje, un profesor asociado del programa que asesora a los docentes en la planificación del desarrollo profesional, y una persona que brinda apoyo administrativo en la gestión de las matrículas de los cursos y mantiene informadas a las facultades. El equipo gestiona la creación de entre tres y cuatro nuevos cursos al año, la revisión de los cursos actuales en un plazo de tres años, la contratación y evaluación del desempeño de los instructores de los cursos OL y el registro y la presentación de informes correspondientes a las más de 1000 matriculaciones de profesores que se producen en un año académico. En estos últimos años, la unidad OFD ha ampliado su misión para brindar apoyo a 150 estudiantes de postgrado al año que desean consolidar y mejorar sus habilidades en la enseñanza online. Desde la creación de la unidad en 2008, se han matriculado más de 5000 profesores y más de 1200 estudiantes de posgrado.

\section{Misión}

Algo esencial para la OFD del World Campus es que las decisiones relacionadas con el plan de estudios, la enseñanza y el presupuesto se basan en la misión de la unidad. La OFD ha pasado de centrar el foco en la asistencia a los cursos, la obtención de certificados y el aumento de las matrículas a tener una visión más a largo plazo: brindar apoyo a los profesores online a lo largo de sus carreras profesionales docentes. Esta perspectiva general está presente en la unidad a la hora de gestionar las matriculaciones, ampliar la oferta de cursos y formar a los profesores de la Pennsylvania State University que imparten los cursos OL, prestando en todo momento atención a la calidad y la sostenibilidad. Esta misión sugiere que cada uno de los miembros del cuerpo docente que completa un curso OL puede convertirse en un socio de enseñanza online a largo plazo. También plantea que, más allá de la competencia, ahora es posible colaborar con personas y grupos docentes para lograr un verdadero dominio de la enseñanza online.

\section{Desarrollo curricular de los cursos OL}

El plan de estudios principal se centra en las competencias básicas. Las materias de los cursos OL incluyen fundamentos de la enseñanza online, introducción al World Campus, problemas de accesibilidad de los alumnos online, conceptos básicos del sistema de gestión del aprendizaje (LMS) (en nuestro caso, Canvas) y manejo del LMS, evaluación de los alumnos online, enseñanza a diversas poblaciones estudiantiles, como estudiantes adultos, militares e internacionales, uso de grupos y equipos en el aprendizaje online y creación de cursos para fomentar la accesibilidad. Pueden surgir ideas para la creación de nuevos cursos OL a partir de varios procesos, como evaluaciones periódicas de las necesidades del profesorado, seguimiento de las tendencias del sector y casualidades durante colaboraciones entre la unidad y los distintos ámbitos académicos. Por ejemplo, socios de otra área de la universidad desarrollaron e impartieron un curso de ludificación. Se creó un curso de enseñanza a estudiantes militares a raíz de la presencia de especialistas militares en el World Campus, mientras que el Curso de Enseñanza a Alumnos Internacionales surgió a partir de una conversación con colegas del Programa de Educación Comparativa e 
Internacional del College of Education. La necesidad de desarrollo profesional de los consejeros dio lugar a un curso de asesoramiento académico (destinado a docentes que orientan a los alumnos). Se originó un curso OL sobre cómo utilizar la biblioteca digital, asistir en conflictos online de los alumnos y realizar investigaciones con estudiantes online porque la unidad quiso dar respuesta a las necesidades de nuestros componentes fundamentales.

Cada uno de los cursos OL y todo el plan de estudios son creados con varios propósitos en mente:

1) Dar a conocer las mejores prácticas de la enseñanza online y hacer que se practique con ellas.

2) Conseguir que el profesorado se familiarice con el entorno de los cursos online, lo que se realiza imitando un curso

3) Ayudar a los profesores a adquirir conocimientos en áreas temáticas importantes que resultan fundamentales para la enseñanza online.

4) Satisfacer las necesidades de formación que presenta la comunidad de capacitación docente de toda la universidad.

El progreso de los cursos es supervisado por la unidad OFD, incluso aunque hayan sido creados por otros expertos en la materia. El proceso de diseño, creación, prueba y lanzamiento oficial suele prolongarse entre dos y tres semestres, o menos si los colaboradores y socios pueden dedicarle más tiempo de lo habitual. Los derechos y acuerdos estándar de propiedad intelectual se aplican a los cursos OL igual que si se tratara de cursos académicos por créditos, pero la unidad OFD tiene la autoridad final para tomar decisiones relacionadas con la creación, revisión 0 interrupción de un curso OL.

Además de elaborar y revisar el programa de los cursos, la OFD agrupa estos últimos en certificados de enseñanza online. El Foundations of Online Teaching Certificate (Certificado de Fundamentos de la Enseñanza Online) es el eje central de un programa compuesto por cuatro cursos, el cual está orientado a la adquisición de conocimientos y competencias esenciales para la impartición de enseñanza universitaria online. El Instructional Practice Certificate (Certificado de Práctica Formativa) incluye cursos OL cuyo objetivo es mejorar la pedagogía y la formación online. El Professional Practice Certificate (Certificado de Práctica Profesional) engloba cursos OL y experiencias diseñados para mejorar la enseñanza a través del desarrollo profesional continuo. El Learning and the Learner Certificate (Certificado de Aprendizaje y Alumno) incluye cursos OL diseñados para enseñar a los profesores las singulares necesidades de aprendizaje de las poblaciones estudiantiles online. El Course Authoring Certificate (Certificado de Creación de Cursos) engloba cursos OL diseñados para ayudar al profesorado a comprender los principios básicos relativos al diseño del espacio de aprendizaje digital. 


\section{Modelo de instructores docentes}

Un componente fundamental del plan de estudios de la OFD son los cursos OL dirigidos por un instructor. Se trata de cursos grupales de 20-25 asistentes (profesores de la Pennsylvania State University que imparten clases online) que tienen una duración de entre cuatro y cinco semanas y son impartidos por un instructor online que está realizando prácticas en esa misma universidad. Este modelo de profesores que enseñan a profesores se basa en tres supuestos:

1) Los profesores, especialmente aquellos que se muestran reacios a matricularse en un curso OL (pero tienen que hacerlo porque así se lo exige su programa 0 facultad), prefieren que les enseñe un colega a que lo haga un instructor sin formación en docencia. Este es el caso especialmente de los docentes que no quieren ser "alumnos de una clase onli$n e "$ ", pero cuyo departamento académico 0 facultad les obliga a formarse.

2) Los profesores que se convierten en instructores de OL ya han obtenido nuestro certificado básico, el Foundations of Online Teaching Certificate. Sin embargo, enseñar a otros profesores es un paso importante para mejorar el desarrollo profesional, el nivel de satisfacción docente y el grado de eficacia en sus propios cursos académicos online.

3) Los profesores que imparten cursos OL provienen de todas las disciplinas, lo que ofrece a los "alumnos" (profesores asistentes) de dichos cursos una gran variedad de experiencias y puntos de vista.
Los instructores de OL reciben apoyo individual del director y el subdirector, incluido un análisis sobre la evaluación del curso (donde se califica tanto el curso como la instrucción) y una reflexión sobre la experiencia de la impartición del curso. Los instructores de OL también pasan a formar parte de la Faculty Learning Community (Comunidad de aprendizaje docente), un espacio virtual del LMS que fomenta la comunicación, el intercambio de información y el debate en torno a la impartición de cursos OL y la enseñanza online en general. En la Faculty Learning Community (algo así como un espacio del curso donde los profesores quedan registrados en el LMS), los instructores de OL pueden conversar de manera asincrónica sobre los desafíos y logros de la enseñanza online, compartir recursos y ejemplos de su experiencia docente, y ofrecer apoyo continuo y conocimientos a sus colegas. Tanto los instructores OL como el director y el subdirector participan en esta comunidad de aprendizaje docente online, estos últimos en calidad de líderes de la unidad OFD.

Los instructores de OL reciben una modesta retribución por sus servicios de enseñanza a través de suplementos salariales de acuerdo con la categoría laboral que ostentan en la universidad. Dado que cada año se necesitan instructores para aproximadamente 45 secciones de cursos OL, cifra que va en aumento año tras año, es necesario incrementar constantemente el presupuesto operativo de la unidad OFD a fin de cubrir las retribuciones de los instructores. La asignación anual de fondos a dicha unidad demuestra el compromiso de la universidad con 
la capacitación docente para que el profesorado aprenda a enseñar online.

\section{Servicios personalizados para programas, facultades y campus}

La mayor parte de los recursos de la OFD se destinan a mantener el programa de los cursos OL y a brindar apoyo a los instructores de estos. Una función secundaria en consonancia con la misión de la unidad es el diseño de servicios presenciales personalizados para programas y facultades. Estos incluyen orientación a nuevo profesorado, presentaciones en reuniones de capacitación docente presencial y online, personalización de certificados de docencia online según los objetivos de la facultad, y colaboraciones con programas académicos para formar y brindar apoyo a nuevos tipos de instructores online. Un ejemplo destacable es una facultad con una gran cartera de programas y cursos online. Los administradores y el cuerpo docente principal de dicha facultad organizaron un taller de tres días en el campus cuyo tema fue la capacitación docente del profesorado online. La facultad corrió con los gastos de desplazamiento de más de 50 instructores online procedentes de todo el país, así como de algunos alumnos virtuales, para que pudieran acudir al campus a hablar sobre la enseñanza y participar en diversas presentaciones sobre las mejores prácticas para la enseñanza y el diseño de cursos online. El liderazgo de la OFD trabajó en el comité de planificación de eventos, presentó ante los asistentes una visión general de los cursos OL y los certificados, y participó en sesiones de grupo sobre mejores prácticas.

\section{Becas e investigación}

Los esfuerzos de investigación de la OFD se centran en determinar la eficacia de los cursos OL. Actualmente, la autonomía académica y la cultura docente impiden que la OFD asista al curso académico de un instructor. Esa sería la mejor manera de observar hasta qué punto el instructor ha aplicado los conceptos y prácticas que aprendió en el curso OL, un indicador fiable de la eficacia. Por ahora, los autoinformes de los instructores y la asistencia de estos a cursos adicionales son indicadores de la eficacia del programa.

\section{Colaboración a nivel de uni- versidad}

La elaboración del plan de estudios, el apoyo para la impartición de cursos OL (y las funciones administrativas relacionadas, como el seguimiento de las matrículas y la comunicación con programas académicos y facultades) forman parte de los servicios de desarrollo profesional personalizados que constituyen la función principal de la OFD, cuyo objetivo es cumplir la misión de dicha unidad: asistir a los docentes online a lo largo de sus carreras profesionales. Otra forma en que la unidad OFD presta servicio es a través de la robusta estructura del comité de la Pennsylvania State University. Los líderes de la OFD participan en varios comités formales permanentes, grupos de trabajo relacionados con el plan estratégico y grupos de proyectos informales donde se congregan las comunidades de capacitación docente y diseño de aprendizaje, las cuales de otro modo apenas tendrían contacto entre sí en una institución compleja compuesta por distintos campus como es 
la Pennsylvania State University. El valor de este servicio pone de manifiesto que los grandes recursos de aprendizaje online a nivel de universidad se aprovechan mejor cuando son coordinados a través de redes formales e informales.

\section{Conclusión}

La unidad de capacitación docente online del World Campus es única entre las grandes y complejas universidades de investigación que destacan en el ámbito online. La unidad sigue evolucionando y ha pasado de desempeñar una función de capacitación docente centrada únicamente en la impartición de cursos OL, a convertirse en un centro de aprendizaje con un robusto programa de cursos de desarrollo profesional, programas personalizados y colaboraciones para la creación de nuevos cursos, como Diseño Universal del Aprendizaje, Pensamiento Integrador y la beca de Enseñanza y Aprendizaje. Aunque se financia a través del reparto de beneficios del World Campus, la unidad está a disposición de todos y cada uno de los profesores de la Pennsylvania State University que precisen sus servicios, independientemente de si imparten clase en el World Campus 0 no. Además, actualmente la unidad es el principal centro de preparación de alumnos de postgrado para la impartición de cursos online en la Pennsylvania State University u otras universidades.

La evolución de la unidad OFD, su función dentro de la universidad y su contribución al campo de la capacitación docente online en general indican que la decisión de hace años de crear la unidad y permitir- le prosperar por medio de una financiación y un respaldo político constantes ayudó a situar la Pennsylvania State University en una posición líder en el ámbito de la educación online. Para las universidades que se inician en el aprendizaje online, puede resultar tentador centrarse en el lanzamiento de cursos y programas de grado y, en cambio, olvidarse del tema de la instrucción. A menudo no se tiene en cuenta que la enseñanza online es distinta de la enseñanza presencial y que requiere capacitación especializada. Resulta comprensible que los educadores con formación tanto reglada como no reglada e inmersos en la instrucción presencial se equivoquen al pensar que sus habilidades en enseñanza pueden aplicarse al entorno online cuando, en la práctica, esto no es lo habitual. Lo que ahora sabemos es que gran parte de la instrucción online puede transferirse al formato presencial, pero no sucede lo mismo al contrario. Se suele pasar por alto el hecho de que, para conseguir un aprendizaje online de calidad, se necesita más que un curso bien diseñado; se requiere instrucción especializada y competente para hacer que un curso cobre vida y para despertar y mantener la participación de los alumnos.

Aquellas universidades que optan por dar importancia e invertir en preparación para la enseñanza online abordan varios problemas críticos de forma simultánea. En primer lugar, la preparación para la enseñanza online hace posible un aumento de la calidad de la experiencia y el éxito del estudiante. Los alumnos que se sienten comprometidos con sus profesores online tienen más probabilidades de perseve- 
rar en sus cursos y grados. Teniendo en cuenta los costes en marketing que acarrea conseguir que un estudiante que se plantea matricularse finalmente lo haga, la capacitación docente online es una pequeña inversión que permite mejorar las probabilidades de retención de alumnos a través del impacto directo de la calidad del instructor. En segundo lugar, la capacitación docente online ayuda a fomentar la sostenibilidad de los programas porque permite al profesorado ampliar su repertorio de recursos de enseñanza, alcanzar el éxito y aumentar la satisfacción en sus clases online y perdurar en el personal a fin de lograr la calidad y reputación propias de un programa académico de grado online. En tercer lugar, una de las funciones de la capacitación docente online consiste en centrarse en la teoría de la enseñanza y el aprendizaje, no solo en pulsar botones y jugar con toda la parafernalia que rodea el espacio de aprendizaje online. A medida que el profesorado vaya adquiriendo conocimientos sobre la enseñanza online (para muchos quizás sea la primera formación reglada en enseñanza que reciben), se irán familiarizando sobre las mejores prácticas y los principios de la enseñanza y el aprendizaje en general. En cuarto lugar, estos conocimientos sobre cómo funciona el aprendizaje online y de qué manera aprenden los alumnos virtuales aporta valor agregado a la enseñanza impartida presencialmente. Una observación común entre los profesores que han recibido formación en enseñanza online es que, una vez que regresan al formato presencial, son más eficaces y están más satisfechos. Esto se debe en parte al aprendizaje de principios educativos esenciales para la enseñanza online, como el diseño de instrucciones claras, la alineación entre los objetivos de aprendizaje de los alumnos, unas tareas adecuadas y unas evaluaciones precisas y un diseño de cursos retrospectivo, donde se comienza por los resultados y se termina por las lecturas, tareas y metodologías de calificación. La preparación para la enseñanza online también puede aumentar el nivel de competencia docente y el grado de seguridad en sí mismo del profesorado, especialmente en el espacio desconocido del aprendizaje asincrónico a distancia.

La intencionalidad de la capacitación docente online, por ejemplo, en cursos oficiales como los cursos OL de la OFD de la Pennsylvania State University, envía varios mensajes claros a aquellas facultades que desean crear programas online con la finalidad de obtener nuevos ingresos y satisfacer a una población estudiantil más amplia:

1) La enseñanza es importante y merece la pena invertir recursos en ella.

2) Una práctica educativa reflexiva y una evaluación eficaz del rendimiento docente son fundamentales para la calidad de la enseñanza online y el éxito de los alumnos.

3) El dominio de la enseñanza online es viable, pero se necesitan años de aprendizaje guiado y práctica.

4) La enseñanza online puede aplicarse al modelo presencial, lo que permite mejorar la enseñanza general en todos sus formatos. 
Dicha iniciativa de formación permanente requiere tiempo para madurar y alcanzar credibilidad en el ámbito universitario, aunque la paciencia parece algo difícil de aplicar dada la velocidad con la que transcurren los cambios que afectan a la educación superior, especialmente la virtual. Sin embargo, en la enseñanza, la calidad no entiende de atajos. La calidad cuesta. La calidad también hace que una iniciativa online sea competitiva. De igual modo que los programas y cursos de grado online se han convertido en la corriente principal, la capacitación docente online también debería convertirse en una función esencial de aquellas universidades que desean ejercer un papel significativo y creíble como proveedores de aprendizaje online.

\section{Referencias bibliográficas}

Allen, I. E. y Seaman, J. (2008). Online Nation: Five Years of Growth in Online Learning. Needham, MA: The Sloan Consortium.

Bigatel, P., Ragan, L., Kennan, S., May, J. y Redmond, B. (2012). The Identifications of Online Teaching Competencies for Teaching Success. Journal of Asynchronous Learning Networks, 16 (1), 59-77.

Chickering, A. y Gamson, Z. (1987). Seven Principles for Good Practice in Undergraduate Education. American Association for Higher Education Bulletin, 3-7. Recuperado de https://bit. ly/2IaL3Ga (Consultado el 08-10-2019).

Diehl, W. (2016). Online instructor and teaching competencies: Literature review for Quality Matters. Quality Matters. Recuperado de https://bit.ly/2mQpBBb (Consultado el 08-102019).

Educause (2017). Key Issues in Teaching and Learning 2017. Recuperado de https://bit.ly/ 2meNLVs (Consultado el 08-10-2019).

Educause (2019). Key Issues in teaching and Learning 2019. Recuperado de https://bit.ly/2lsyM3E (Consultado el 08-10-2019).
Gregory, R. y Martindale, T. (2016). Faculty Development for Online Instruction in Higher Education. Association for Educational Communication and Technology. Recuperado de https:// bit.ly/2ouzvso (Consultado el 08-10-2019).

Horvitz, B., Beach, A., Anderson, M. y Xia, J. (2015). Examination of faculty self-efficacy related to online teaching. Innovative Higher Education, 40 (4), 305-316.

McQuiggan, C. (2012). Faculty Development for Online Teaching as a Catalyst for Change. Journal of Asynchronous Learning Networks, 16 (2), 27-61. Recuperado de https://bit.ly/2nPBffx (Consultado el 08-10-2019).

Palloff, R. M. y Pratt, K. (2011). The Excellent Online Instructor: Strategies for Professional Development. San Francisco: Jossey-Bass.

The Pennsylvania State University (2019). Faculty Competencies. Recuperado de https://bit.ly/ 2mi9lbN (Consultado el 08-10-2019).

\section{Biografía del autor}

Laurence B. Boggess es Doctor en Teoría y Política Educativa por la Pennsylvania State University y Licenciado en Educación, Política y Organizaciones por el Peabody College de Educación de la Vanderbilt University. Dirige la unidad de capacitación docente online del Penn State World Campus e imparte clases en la Facultad de Liderazgo Educativo para el programa de ciencias de la educación online. Algunos de los temas de sus investigaciones son la enseñanza y el aprendizaje online, la capacitación docente online, los distritos escolares urbanos y el liderazgo educativo.

(D) https://orcid.org/0000-0003-0378-1060 


\section{revista española de pedagogía} año 78, n² 275, enero-abril 2020

Spanish Journal of Pedagogy

year 78, n. 275, January-April 2020

\section{Sumario*}

\section{Table of Contents **}

\section{Cuestiones de enseñanza de las matemáticas}

Wendolyn Elizabeth Aguilar-Salinas, Maximiliano de las Fuentes-Lara, Araceli Celina Justo-López y Ana Dolores Martínez-Molina

Instrumento de medición para diagnosticar las habilidades algebraicas de los estudiantes en el Curso de Cálculo Diferencial en ingeniería

A measurement instrument for establishing the algebraic skills of engineering students on a Differential Calculus Course in engineering

Maria Burgos, Pablo Beltrán-Pellicer y Juan D. Godino La cuestión de la idoneidad de los vídeos educativos de matemáticas: una experiencia de análisis con futuros maestros de educación primaria

The issue of didactical suitability in mathematics educational videos: experience of analysis with prospective primary school teachers

\section{La formación online en el mundo de la educación: experiencias de los Estados Unidos}

Gerald LeTendre y Tiffany Squires

Integración de programas de máster online y presenciales en educación

Integrating online and residential master's programs in education

\section{Laurence B. Boggess}

Innovación en la capacitación docente online: un modelo organizacional para brindar apoyo a largo plazo a la docencia online Innovations in online faculty development: an organizational model for long-term support of online faculty

Brian Redmond

Liderazgo de equipo compartido de un programa online

27 Shared team leadership for an online program

\footnotetext{
* Todos los artículos están también publicados en inglés en la página web de la revista: https://revistadepedagogia.org.

** All the articles are also published in English on the web page of the journal: https://revistadepedagogia.org.
} 


\section{Estudios y Notas}

\section{Paolo Scotton}

Pensar en común, vivir en plenitud. La experiencia de

la filosofía con los niños y niñas

Thinking together, living fully. Experiencing philosophy with

children

103

\section{Manuel Delgado-Garcia, Sara Conde Vélez y Ángel}

Boza Carreño

Perfiles y funciones del tutor universitario y sus

efectos sobre las necesidades tutoriales del alumnado

Profiles and functions of university tutors and their effects on

students' tutorial needs

119

\section{Reseñas bibliográficas}

Luri, G. (2019). La imaginación conservadora: una defensa apasionada de las ideas que han hecho del mundo un lugar mejor (Enrique Alonso Sainz).

Quigley, C. F. y Herro, D. (2019). An educator's guide to STEAM. Engaging students using real-world problems (Juan Luis Fuentes).Prince, T. (2019). Ejercicios de mindfulness en el aula. 100 ideas prácticas (José V. Merino Fernández).

\section{Informaciones}

Francisco Altarejos: la filosofía de la educación hecha vida (Concepción Naval); World Educational Research Association Focal Meeting 2020: «Networking Education: Diverse Realities, Common Horizons»);

Conclusiones del IX Congreso Internacional de Filosofía de la Educación; Ebook 2019: José Antonio Ibáñez-Martín (Ed.) (2019). La misión de las revistas de investigación en el mundo educativo. The mission of research journals in the world of education.

\section{Instrucciones para los autores}

Instructions for authors

\section{Solicitud de originales}

Call for papers

169

XV Congreso Internacional de Teoría de la Educación

XV International Congress on Theory of Education

ISSN: 0034-9461 (Impreso), 2174-0909 (Online)

https://revistadepedagogia.org/

Depósito legal: M. 6.020 - 1958

INDUSTRIA GRÁFICA ANZOS, S.L. Fuenlabrada - Madrid 


\section{Innovations in online faculty development: an organizational model for long-term support of online faculty}

\section{Innovación en la capacitación docente online: un modelo organizacional para brindar apoyo a largo plazo a la docencia online}

Laurence B. BOGGESS, PhD. Director. The Pennsylvania State University (Ibb150@psu.edu).

\section{Abstract:}

In 2008, Penn State University's online delivery organization, Penn State World Campus, established a dedicated faculty development unit to improve online instruction. The Online Faculty Development (OFD) unit was designed to prepare faculty for online teaching through a large and varied curriculum of asynchronous online training courses built on the theory and research of best practices for online instruction. This paper describes the Penn State World Campus OFD unit, its mission and program, and the assumptions underlying its day-to-day operations.

Keywords: online faculty development, online teaching, professional development, online teaching competencies, asynchronous training, online teaching certificate.

\section{Resumen:}

En 2008, la organización de enseñanza online de la Pennsylvania State University, el Penn State World Campus, creó una uni-

Revision accepted: 2019-07-18.

This is the English version of an article originally printed in Spanish in issue 275 of the revista española de pedagogía. For this reason, the abbreviation EV has been added to the page numbers. Please, cite this article as follows: Boggess, L. B. (2020). Innovación en la capacitación docente online: un modelo organizacional para brindar apoyo a largo plazo a la docencia online | Innovations in online faculty development: an organizational model for long-term support of online faculty. Revista Española de Pedagogía, 78 (275), 73-87. doi: https://doi.org/10.22550/REP78-1-2020-01 
dad dedicada a la capacitación docente para mejorar la formación online. La unidad de capacitación docente online (en adelante OFD, del inglés Online Faculty Development) fue diseñada con el objetivo de preparar al profesorado para la enseñanza online a través de un programa amplio y variado de cursos asíncronos de formación online basados en la teoría e investigación de las mejores prácticas relacionadas con la instrucción online. En este documento se describe la unidad OFD del Penn State World Campus, su misión y programa, y las premisas sobre las que se basan sus operaciones diarias.

Descriptores: capacitación docente onli$n e$, enseñanza online, desarrollo profesional, competencias de enseñanza online, formación asíncrona, certificado de enseñanza online.

\section{Introduction}

Online education has become a mainstream delivery system for academic degree programs and courses in higher education. Online teaching, however, can be a very different experience to face-to-face teaching because the learning environment - asynchronous, distant, and digital - is still new for many instructors transitioning from the face-to-face classroom. Online teaching requires specialized training designed to develop best practices, understandings, skills, and competencies needed for effective instruction and student success. This is the case for several reasons:

1) Few skills and competencies from face-to-face teaching transfer to online.

2) Many mid-career instructors new to online teaching lack a first-hand understanding of how online students learn.
3) Online course design and teaching require a familiarity of learning theory unfamiliar to many instructors, for example, how to align student learning outcomes, assessments, and assignments, design effective online discussion questions or facilitate effective online group projects.

For many instructors, face-to-face and online, online faculty development training is their first formal introduction to teaching. According to the Educause Learning Initiative, a thought leader in higher education in the United States, the number one issue in teaching and learning in 2017 and 2019 was faculty development (Educause, 2017, 2019). With online learning here to stay, universities should now be turning their attention to preparing faculty to teach online with high levels of competence and quality. This paper describes the theory and practice behind one university's recognition that online courses need to be taught by 
specifically trained instructors in order to improve and maintain quality, assure enrollment growth and student success, and solidify institutional brands through quality instruction.

Online faculty development (OFD) is a recently-emerging subset of higher education faculty development with a growing body of research to support its importance in online education and its best practices (for example, Allen \& Seaman, 2008; Bigatel, Ragan, Kennan, May, \& Redmond, 2012; Gregory \& Martindale, 2016; McQuiggan, 2012; Palloff \& Pratt, 2011). OFD accepts core assumptions about effective teaching and successful learning online:

1) While «good teaching is good teaching,» there are specific skills, understandings, and competencies required for online, asynchronous instruction that are not reliably intuitive and self-teaching for a face-to-face instructor new to online teaching.

2) Few face-to-face teaching techniques translate to the online space while many online practices positively inform and enhance face-to-face teaching.

3) Research is becoming settled on best practices for effective online instruction and student engagement.

4) Institutional commitment to OFD, including a dedicated and expert staff, consistent funding, and a university-wide long-term plan, is essential for preparing instructors for the present and future of higher education online learning.

Further, as a professional development practice, OFD is driven by and responsive to innovation. In American universities, faculty development for face-to-face teaching is rarely required of newly-minted assistant professors and newly-hired adjunct instructors. Higher education classroom instruction has a long history of assuming that subject area experts are teaching experts as well, a false assumption that can lead to ineffective instruction. In contrast, the movement among universities to require some type of formal preparation for online teaching is a major innovation in U.S. higher education. Pre-teaching training for online instruction is motivated by several factors:

1) The recognition that the asynchronous digital learning space is sufficiently different from the face-to-face classroom as to require specialized training.

2) The allocation of resources made available to the «team approach» of moving face-to-face courses and programs online.

3) The promise of new revenue through online enrollments.

4) The possibility of higher college rankings via extensive training to teach online.

With the normalization of training and credentialing for online instructors, we see a trend affirming the relationship between educational professional 
development, quality instruction, and student success.

\section{Best practices for online ins- truction}

Chickering and Gamson (1987) identified seven principles for successful undergraduate teaching. They are expressed as instructor behaviors, identifiable as competencies and understandings:

1. Encourage contact between students and faculty.

2. Develop reciprocity and cooperation among students.

3. Encourage active learning.

4. Give prompt feedback.

5. Emphasize time on task.

6. Communicate high expectations.

7. Respect diverse talents and ways of learning.

These are not aspirational; each of the seven behaviors can be enacted with planning, guided training, practice, and a purpose intending to positively impact student learning and success. Underlying these behaviors are several key factors:

1) The importance of building relationships through teaching and learning.

2) An instructor positionality of «student-centeredness».
3) The value of student input into course design and management.

4) The need for tight alignment among the core components of a course: student learning objectives, assignments, assessments, and course design.

5) The importance of student engagement with the instructor, peers, and the course content and curriculum.

In online higher education instruction, these behaviors are still relevant but must be conceptualized and operationalized for a different demographic and learning space: the adult online learner and the traditional college age student learning asynchronously, in multiple time zones, and through a digital interface. The Chickering and Gamson (1987) instructor behaviors translated to online must leverage the affordances of online learning and innovate around the constraints inherent in the asynchronous digital space. For example, «encourage contact between students and faculty» and «respect diverse talents and ways of learning," can be more productive online because, unlike the face-to-face lecture or discussion, every student voice is heard in discussion forums. Issues of race, ethnicity, class and physical accessibility are somewhat neutralized online, allowing for increased equality of contribution and sharing of diverse points of view and life experiences.

Research building on Chickering and Gamson found that these face-to-face 
TABLE 1. Online Teaching Competencies.

\begin{tabular}{|c|c|c|}
\hline Pedagogical & Technical & Administrative \\
\hline $\begin{array}{l}\text { Attend to the unique } \\
\text { challenges of distance } \\
\text { learning where learners } \\
\text { are separated by time } \\
\text { and geographic proximi- } \\
\text { ty and interactions are } \\
\text { primarily asynchronous } \\
\text { in nature. }\end{array}$ & $\begin{array}{l}\text { Complete basic computer } \\
\text { operations. }\end{array}$ & $\begin{array}{l}\text { Log-in to the course and acti- } \\
\text { vely participate. }\end{array}$ \\
\hline $\begin{array}{l}\text { Be familiar with the } \\
\text { unique learning needs } \\
\text { and situations of both } \\
\text { traditional age and adult } \\
\text { learners, providing an } \\
\text { educational experience } \\
\text { that is appropriate for } \\
\text { both. }\end{array}$ & $\begin{array}{l}\text { Successfully log into the } \\
\text { LMS and access the course. }\end{array}$ & $\begin{array}{l}\text { Communicate to students } \\
\text { when assignments and exams } \\
\text { will be graded and returned. }\end{array}$ \\
\hline $\begin{array}{l}\text { Have mastery of course } \\
\text { content, structure, and } \\
\text { organization. }\end{array}$ & $\begin{array}{l}\text { Successfully navigate the } \\
\text { course space. }\end{array}$ & $\begin{array}{l}\text { Provide a comprehensive } \\
\text { syllabus that adheres to insti- } \\
\text { tutional syllabus policy. }\end{array}$ \\
\hline $\begin{array}{l}\text { Respond to student } \\
\text { inquiries. }\end{array}$ & $\begin{array}{l}\text { Set-up and manage student } \\
\text { grades. }\end{array}$ & $\begin{array}{l}\text { Mediate course-related student } \\
\text { conflicts. }\end{array}$ \\
\hline $\begin{array}{l}\text { Provide detailed feed- } \\
\text { back on assignments and } \\
\text { exams. }\end{array}$ & $\begin{array}{l}\text { Effectively use course com- } \\
\text { munication systems. }\end{array}$ & $\begin{array}{l}\text { Adhere to the institutional } \\
\text { policies regarding student } \\
\text { educational rights, privacy and } \\
\text { accessibility. }\end{array}$ \\
\hline $\begin{array}{l}\text { Communicate with } \\
\text { students about course } \\
\text { progress and changes. }\end{array}$ & Manage the course roster. & $\begin{array}{l}\text { Revise course content and } \\
\text { instructional materials based } \\
\text { on student feedback. }\end{array}$ \\
\hline $\begin{array}{l}\text { Promote and encourage } \\
\text { a learning environment } \\
\text { that is safe, inviting and } \\
\text { mutually respectful. }\end{array}$ & $\begin{array}{l}\text { Manage student submis- } \\
\text { sions. }\end{array}$ & $\begin{array}{l}\text { Know where and when to } \\
\text { get technical assistance and } \\
\text { support for you and your } \\
\text { students. }\end{array}$ \\
\hline $\begin{array}{l}\text { Monitor and manage } \\
\text { student progress. }\end{array}$ & $\begin{array}{l}\text { Manage the course files } \\
\text { and folders within the LMS } \\
\text { (when appropriate). }\end{array}$ & $\begin{array}{l}\text { Communicate expectations of } \\
\text { student course behavior. }\end{array}$ \\
\hline
\end{tabular}




\begin{tabular}{|l|l|l|}
\hline $\begin{array}{l}\text { Communicate course } \\
\text { goals and outcomes. }\end{array}$ & $\begin{array}{l}\text { Be aware of, inform stu- } \\
\text { dents about, and monitor } \\
\text { compliance to institutional } \\
\text { academic integrity policies. }\end{array}$ \\
\hline $\begin{array}{l}\text { Provide evidence to } \\
\text { students of their pre- } \\
\text { sence on the course on a } \\
\text { regular basis. }\end{array}$ & $\begin{array}{l}\text { Report grades to students and } \\
\text { record grades to the Universi- } \\
\text { ty's grading system as requi- } \\
\text { red. }\end{array}$ \\
\hline $\begin{array}{l}\text { Demonstrate sensitivity } \\
\text { to disabilities and diver- } \\
\text { sities including aspects } \\
\text { of cultural, cognitive, } \\
\text { emotional, and physical } \\
\text { differences. }\end{array}$ & \\
\hline
\end{tabular}

Fuente: The Pennsylvania State University, 2019.

best practices could be translated to online teaching best practices, generating multiple discrete skills and understandings relevant to asynchronous learning. Bigatel et al. (2012) confirmed three categories of online instructor competencies encompassing online teaching: pedagogical competencies, technological competencies, and administrative competencies (see Table 1). Each of these best practices was identified as having a positive association with student engagement and a reliable measure of student learning and success.

Diehl (2016) further includes instructional design as a teaching-related competency and updates the research on online teaching competency and the standards movement for online instruction for Quality Matters, a rubric-based standard for online course design.
While these best practices maintain an association with effective face-to-face instruction, the factors of distance, asynchronicity, rapidly changing technology, and demographics of online learners suggest the need for specialized training for the online instructor. For that purpose, Penn State established a dedicated faculty development unit in the World Campus organizational structure responsible for supporting all Penn State faculty who teach online. This initial support resulted in the development of a curriculum of online training courses made available to faculty free of charge. Along the way, the descriptor «online» was added to «faculty development» to distinguish the specific learning experiences necessary to develop the skills, understandings, and competencies necessary for effective online instruction. 


\section{A dedicated unit for online fa- culty development at penn state}

The Online Faculty Development (OFD) unit of Penn State World Campus was established in 2008 to offer online, asynchronous courses designed to help Penn State faculty develop understandings and competencies in online teaching. OFD is a unit within the World Campus Academic Affairs organization along with Learning Design, Program Planning and Management, Academic Advising, Student Affairs, and Outreach Information and Technology. Established in the early days of World Campus and approved by the governance of Penn State Outreach as a formal support for quality online instruction, the OFD unit is funded through a tuition revenue share between World Campus (the platform provider) and the academic program and its college (the content and instruction provider).

One of the unique design features of Penn State's online initiative is the division of responsibilities between World Campus and the university's colleges. World Campus provides the online platform and technical support for the colleges to offer their academic degree programs. World Campus has no authority over the academic content, hiring, performance assessment of faculty, or course assignments of a program's faculty. World Campus can advise an academic program seeking to establish online degrees and can help market those programs, but retains only a support role in academic quality. The relationship between Penn State World Campus and the colleges can be thought of as a decentralized design, with the colleges retaining their academic authority. All World Campus courses are authored and taught by Penn State faculty. This design means that all degrees earned online through World Campus say «Penn State», and not «Penn State World Campus.»

Given this organizational context, World Campus Online Faculty Development has been given the support and freedom to be an innovative, researchedbased leader for online teaching improvement across the university as well as contributing to the specialized field of higher education professional development. All of the unit's research-based online training courses, customized services, and one-on-one work with faculty is offered free of charge to programs and individual professors.

The Online Faculty Development (OFD) unit at Penn State World Campus offers all Penn State University faculty and graduate students a curriculum of over nearly 20 faculty training courses (designated as «OL courses»). Half of the curriculum consists of self-directed courses that take three to five hours to complete. The rest consists of four or five-week cohort-based, instructor-led courses designed to mimic an online academic course with graded assignments and due dates around topics related to best practices for online teaching. The courses take between three to five hours a week to complete and are taught by current Penn State faculty who have earned the World Campus OFD Foundations of Online Teaching Certificate. 
The theory of action behind the OL course design is based on the idea that the best way for an instructor to learn to teach online is to gain knowledge and competencies through learning online. In an OL course, the faculty member, especially one new to online education, becomes a "student», gaining familiarity with the LMS, experiencing the social learning advantages of asynchronous discussions, understanding the online student perspective, and acquiring knowledge and expertise in teaching skills. In addition to content learning, OL course evaluations consistently report that faculty who start off a course resistant, fearful, or insecure about online teaching conclude the course with self-reports of increased confidence and competence, key factors in online teaching self-efficacy (Horvitz, Beach, Anderson, \& Xia, 2015).

\section{World campus ofd: organizatio- nal structure and scope}

In the early 2000's, World Campus and university leadership understood that in establishing and growing an online initiative, it was necessary to make sure that faculty received formal and scaffolded preparation to teach in the new digital learning spaces in online education. The OFD unit was positioned inside the World Campus organizational structure rather than in a college or the Provost's office because it was designated as the primary support of faculty who taught courses offered through the World Campus. As part of the tuition revenue share between World Campus and the colleges, OFD was considered one of the services provided along with the student help desk support, learning design, undergraduate academic advising, IT support, and marketing. In recent years, services have expanded to include online student affairs.

All faculty who teach online at Penn State, either through World Campus degree programs or college-level webbased courses, receive training in online instruction. While some type of training is required of all faculty, the sources of training vary. Training can be provided at the academic program level, from within a college faculty development office, or through the World Campus Online Faculty Development unit. Increasingly in recent years, colleges and academic programs have mandated that their faculty who teach online be trained through OFD's OL courses.

Word Campus offers over 160 degree and certificate programs to 20,000 fully online students. In any given semester, approximately 1,200 Penn State instructors teach courses through World Campus. OFD's mission is to offer all instructors who teach trough World Campus initial preparation and ongoing instructional development. Despite this large scope, OFD is designed to be lean. The unit consists of four people with specific responsibilities: a director with a faculty appointment who oversees curriculum and OL course instructors, an assistant director with a doctorate and learning design experience, a program associate who advises faculty on professional de- 
velopment planning, and an administrative support person who manages course enrollments and reporting back to colleges. The team manages the development of three to four new courses a year, the revision of current courses on a threeyear timeline, the hiring and performance assessment of OL instructors, and the registration and reporting of over 1,000 faculty enrollments in an academic year. In recent years, the OFD unit has expanded its mission to support 150 graduate students a year who seek to establish and improve their online teaching skills. Since the unit's establishment in 2008, there have been over 5,000 faculty enrollments and 1,200 graduate student enrollments.

\section{Mission}

Fundamental to the World Campus OFD organizational unit is curricular, instructional, and budget decisionmaking informed by mission. The unit has evolved from focusing on coursetaking, certificate-earning, and growing course enrollments to a longer-term vision: to support online faculty throughout their teaching careers. This big picture perspective is central to how the unit manages enrollments, expands course offerings, trains the Penn State faculty who teach the OL courses, and balances all of this with attention to quality and sustainability. The mission suggests that each individual faculty member who completes an OL course is potentially a long-term partner in his or her online teaching career. The mission also suggests that beyond competence, it is now possible to work with individuals and groups of faculty for genuine online teaching mastery.

\section{Curriculum development of $\mathrm{OL}$ courses}

The core curriculum focuses on basic competencies. OL course topics include essentials of online teaching, introduction to the World Campus, accessibility issues for online learners, Learning Management System (LMS) basics - in our case Canvas - and operating the LMS hands-on, assessment of online learners, teaching various student populations such as the adult learner, military learner, and the international learner, using groups and team in online learning, and course-authoring for accessibility. Ideas for new OL courses are created through various processes such as periodic faculty needs assessments, monitoring industry trends, and serendipities of cross-unit and academic collaborations. For example, a gamification course was developed and taught by partners in another area of the university. A teaching the military learner course originated with the military specialists in World Campus, and the Teaching the International Learner Course arose out of conversation with colleagues in the College of Education's Comparative and International Education Program. A course on academic advising (for faculty who advise) arose from the need for professional development for advisers. An OL course on using the online library, helping online students crisis, and conducting research with online students all came about because the 
unit was responsive to the needs of our core constituents.

Individual OL courses and the whole curriculum are created with several purposes in mind:

1) To provide exposure to and practice with best practices in online instruction.

2) To familiarize faculty with the online course environment by mimicking an academic course in the OL course design and by treating faculty as students in the OL course with assignments to complete through each week.

3) To help faculty gain knowledge in important topic areas essential to online teaching.

4) To fulfill training needs in the faculty development community across the university.

Course development is supervised by the OFD unit, even when other subject area experts author the course. The process from design, authoring, piloting, and official launching usually takes two to three semesters or less if collaborators and partners can provide additional capacity. Standard intellectual property rights and agreements apply to OL courses as if they were for-credit academic courses, but the OFD unit has final decision-making authority over the creation, revision, or discontinuation of an OL course.
In addition to developing and maintaining a curriculum of courses, OFD bundles courses into online teaching certificates. The Foundations of Online Teaching Certificate is the four-course centerpiece of the certificate curriculum and focuses on developing understandings and competencies essential for online higher education instruction. The Instructional Practice Certificate includes OL courses aimed at improving pedagogy and online instruction. The Professional Practice Certificate clusters OL courses and experiences designed to improve teaching through ongoing professional development. The Learning and the Learner Certificate includes OL courses designed to teach faculty about the unique learning needs of online student populations. The Course Authoring Certificate bundles OL courses designed to help faculty understand the design fundamentals of the digital learning space.

\section{Model of faculty instructors}

An essential component of the OFD curriculum is instructor-led OL courses. These are courses of 20-25 participants (Penn State faculty who teach online) in a four- or five-week cohort model, taught by a currently practicing Penn State online instructor. This model of faculty teaching faculty is based on three assumptions:

1) Faculty, especially those who reluctantly enrolled in an OL course (but are required to by their program or college), prefer to be taught by a colleague rather than a non-faculty educational 
developer. This is especially true for faculty who don't wish to be «students in an online class» but are required to gain training by their academic department or college.

2) Faculty who become OL instructors have already earned our basic Foundations of Online Teaching Certificate. However, teaching other faculty is an important step in their own professional development and teaching satisfaction as well as their effectiveness in their own online academic courses.

3) Faculty who teach OL courses come from all disciplines, providing OL course «students» (faculty participants) a broad range of experience and perspective.

OL instructors receive individual support from the Director and Assistant Director, including a discussion around the course evaluation (rating both the course and the instruction) and reflection on the experience of teaching the course. OL instructors also become part of a Faculty Learning Community, a virtual space in the LMS for communication, information sharing, and discussion around OL teaching and online teaching in general. In the Faculty Learning Community space - much like a course space with faculty enrolled in the LMS, OL instructors can chat asynchronously about challenges and successes in online teaching, share resources and examples of their teaching, and provide their colleagues with ongoing support and expertise. Both the director and the assistant director participate in this online Faculty Learning Community as leaders of the OFD unit as well as OL instructors themselves.

OL instructors are modestly compensated for their teaching through pay supplementals according to their employment status at the university. With approximately 45 sections of OL courses needing instructors each year, and increasing every year, the unit-level operating budget for OFD requires a growing line item for OL instructor compensation. It is a testament to the university's commitment to preparing faculty to teach online that these funds are allocated to the unit each year.

\section{Customized services to pro- grams, colleges, and campuses}

Most of OFD's capacity is allocated toward maintaining the OL course curriculum and supporting the OL course instructors. A secondary function that fulfills the mission is the design of customized, face-to-face services to programs and colleges. These efforts include new faculty orientations, presentations at face-to-face and online faculty development meetings, individualizing certificates based on a college's goals for their online faculty, and working with academic program leads to train and support new types of online instructors. One remarkable example is a college with a large online portfolio of programs and courses. Administrators and lead faculty in this college created an on-campus, three-day workshop around faculty development for online faculty. The 
college funded travel for over 50 online instructors nation-wide and some online students to gather on campus in order to talk about teaching and participate in presentations around best practices for online teaching and course design. OFD leadership worked on the event planning committee, presented an overview of OL courses and certificates to the whole group, and participated in breakout sessions on best practices.

\section{Scholarship and research}

The focus of OFD's research efforts is to determine the effectiveness of OL courses. At this point in time, academic autonomy and faculty culture prevent OFD from sitting in on an instructor's academic course. This would be the best way to observe the extent to which the instructor applied concepts and practices learned in the $\mathrm{OL}$ course - a credible measure of effectiveness. For now, instructor self-reports and additional course-taking are measures of program effectiveness.

\section{Cross-university collaboration}

Development of curriculum and support for instruction of OL courses (and related administrative functions such as enrollment tracking and reporting back to academic programs and colleges) are part of the customized professional development services that constitute OFD's main function to serve the mission of supporting online faculty throughout their careers. Another way the OFD unit serves is through Penn State's robust committee structure. OFD leaders serve on multi- ple formal standing committees, working groups related to the strategic plan, and on informal project groups that bring together the faculty development and learning design communities who would normally be siloed in a complex and multi-campus institution such as Penn State. The value of this service recognizes the vast online learning resources throughout the university are best used when coordinated through formal and informal networks.

\section{Conclusion}

The World Campus Online Faculty Development unit is unique among large and complex Research universities with a robust online initiative. The unit continues to evolve from a faculty development function focused only on OL course completion to a learning center with a robust curriculum of professional development courses, customized programs, and collaborations on new courses such as Universal Design for Learning, Integrative Thinking, and the Scholarship of Teaching and Learning. While funded through the World Campus revenue share, the unit serves any and all interested Penn State faculty, regardless of whether they teach through the World Campus or not. Additionally, the unit is now the main source of preparing graduate students to teach online for Penn State and their future universities.

The evolution of the OFD unit, its function within the university, and its contribution to the field of online faculty development in general suggest 
that the decision years ago to create the unit and allow it to prosper through ongoing funding and political support helped position Penn State as a leader in online education. It can be tempting for universities new to online learning to focus on launching courses and degree programs while overlooking instruction. It is not unusual to miss the fact that teaching online is distinct from face-to-face teaching and requires specialized training. It is understandable that educators trained formally or informally and steeped in face-to-face instruction are mistaken when they believe their teaching skills translate to the online environment when, in practice, few do. What we now know is online instruction has significant transfer to face-to-face instruction, but less so the reverse. It can be easy to overlook the fact that quality online learning needs more than a well-designed course; it needs skilled and competent instruction to make a course come alive and to spark and sustain student engagement.

The university that chooses to emphasize and invest in preparation for online instruction addresses several critical issues simultaneously. One, preparation for online teaching makes possible a greater quality of student experience and success. Students who feel more engaged with their online professors may be more likely to persist through the course and their degree. Given the marketing cost of bringing a student from the prospect stage to the enrollment stage, online faculty development is a small investment to improve the chances of student retention through the direct impact of instructor quality. Two, online faculty development helps build sustainability of a program as faculty expand their teaching repertoire, achieve success and satisfaction in their online teaching, and remain on staff in order to build the quality and reputation of an online academic degree program. Three, an intentional online faculty development effort focuses on teaching and learning theory, not just pushing buttons and playing with the bells and whistles in the online learning space. As faculty learn about teaching online, for many perhaps their first formal instruction in how to teach, they are learning about best practices and principles of teaching and learning in general. Four, this knowledge on how online learning works and how online learners learn brings value added back to the face-to-face classroom. A common observation from those trained to teach online is that they become more effective and satisfied teachers in face-to-face classrooms. This is due in part to learning educational principles essential to online instruction such as designing clear instructions, aligning student learning objectives with relevant assignments and accurate assessments, and backward course design starting with outcomes and ending with readings, assignments, and grading methodologies. Preparation for online teaching can also result in greater degrees of faculty competency for and confidence in teaching, especially in the unfamiliar space of asynchronous distance learning. 
The intentionality of online faculty development training, for example in formal courses such as Penn State's OFD OL courses, sends clear messages to colleges wishing to create online programs to capture new revenue and serve a wider student population:

1) Teaching is important and worth investing resources.

2) Reflective instructional practice and meaningful assessment of teaching performance are critical to online quality and student success.

3) Online teaching mastery is achievable but takes years of guided learning and practice.

4) Online teaching informs face-to-face teaching, improving overall instruction in all delivery modes. Such a permanent training initiative requires time to mature and achieve credibility within a university, and the patience for this may seem unrealistic given the velocity of change - especially in online education - that higher education is experiencing now.

However, as with all teaching, corners cannot be cut to achieve quality. Quality costs. Quality also makes an online initiative competitive. As online degree programs and courses have become mainstream, so should online faculty development as a core function of the university seeking a credible status meaningful purpose as an online learning provider.

\section{References}

Allen, I., \& Seaman, J. (2008). Online Nation: Five Years of Growth in Online Learning. Needham, MA: The Sloan Consortium.

Bigatel, P., Ragan, L., Kennan, S., May, J., \& Redmond, B. (2012). The Identifications of Online Teaching Competencies for Teaching Success. Journal of Asynchronous Learning Networks, 16 (1), 59-77.

Chickering, A., \& Gamson, Z. (1987). Seven Principles for Good Practice in Undergraduate Education. American Association for Higher Education Bulletin, 3-7. Retrieved from https://bit.ly/2IaL3Ga (Consulted on 2019-10$08)$.

Diehl, W. (2016). Online instructor \& teaching competencies: Literature review for Quality Matters. Quality Matters. Retrieved from https://bit.ly/2mQpBBb (Consulted on 2019-10$08)$.

Educause (2017). Key Issues in Teaching and Learning 2017. Retrieved from https://bit.ly/ 2meNLVs (Consulted on 2019-10-08).

Educause (2019). Key Issues in Teaching and Learning 2019. Retrieved from https://bit. ly/2lsyM3E (Consulted on 2019-10-08).

Gregory, R., \& Martindale, T. (2016). Faculty development for Online Instruction in Higher Education. Association for Educational Communication \& Technology. Retrieved from https://bit.ly/2ouzvso (Consulted on 2019-1008).

Horvitz, B., Beach, A., Anderson, M., \& Xia, J. (2015). Examination of faculty self-efficacy related to online teaching. Innovative Higher Education, 40 (4), 305-316.

McQuiggan, Carol. (2012). Faculty Development for Online Teaching as a Catalyst for Change. Journal of Asynchronous Learning Networks, 16 (2), 27-61. Retrieved from https:// bit.ly/2nPBffx (Consulted on 2019-10-08).

Palloff, R. M., \& Pratt, K. (2011). The Excellent Online Instructor: Strategies for Professional Development. San Francisco: Jossey-Bass.

The Pennsylvania State University (2019). Faculty Competencies. Retrieved from https://bit. ly/2mi9lbN (Consulted on 2019-10-08). 


\section{Author's biography}

Laurence B. Boggess holds a PhD in

Educational Theory and Policy from Penn State University and an M. Ed. in Leadership, Policy, and Organizations from Vanderbilt University's Peabody College of Education. He directs the Online Faculty Development unit at Penn State World Campus and teaches on the Educational Leadership faculty for the online MEd program. His research interests include online teaching and learning, online faculty development, urban school districts, and educational leadership.

(iD https://orcid.org/0000-0003-0378-1060 


\section{revista española de pedagogía año 78, n 275, enero-abril 2020 \\ Spanish Journal of Pedagogy \\ year 78, n. 275, January-April 2020 \\ Table of Contents \\ Sumario}

\section{Mathematics teaching} issues

Cuestiones de enseñanza de las matemáticas

Wendolyn Elizabeth Aguilar-Salinas, Maximiliano de las Fuentes-Lara, Araceli Celina Justo-López, \& Ana Dolores Martínez-Molina

A measurement instrument for establishing the algebraic skills of engineering students on a Differential Calculus Course in engineering Instrumento de medición para diagnosticar las habilidades algebraicas de los estudiantes en el Curso de Cálculo Diferencial en ingeniería

Maria Burgos, Pablo Beltrán-Pellicer, \& Juan D. Godino The issue of didactical suitability in mathematics educational videos: experience of analysis with prospective primary school teachers La cuestión de la idoneidad de los vídeos educativos de matemáticas: una experiencia de análisis con futuros maestros de educación primaria

27
Online training in the world of education: experiences from the United States La formación online en el mundo de la educación: experiencias de los Estados Unidos

Gerald LeTendre, \& Tiffany Squires

Integrating online and residential master's programs in education

Integración de programas de máster online y presenciales en educación

\section{Laurence B. Boggess}

Innovations in online faculty development: an organizational model for long.term support of online faculty

Innovación en la capacitación docente online: un modelo organizacional para brindar apoyo a largo plazo a la docencia online

Brian Redmond

Shared team leadership for an online program Liderazgo de equipo compartido de un programa online 
Studies and Notes

Estudios y Notas

\section{Paolo Scotton}

Thinking together, living fully. Experiencing philosophy

with children

Pensar en común, vivir en plenitud. La experiencia de la

filosofía con los niños y las niñas

103

Manuel Delgado-Garcia, Sara Conde Vélez, \& Ángel

\section{Boza Carreño}

Profiles and functions of university tutors and their

effects on students' tutorial needs

Perfiles y funciones del tutor universitario y sus efectos sobre

las necesidades tutoriales del alumnado

119
Book reviews

Luri, G. (2019). La imaginación conservadora: una defensa apasionada de las ideas que han hecho del mundo un lugar mejor [The conservative imagination: A passionate defence of ideas that have made the world a better place] (Enrique Alonso Sainz). Quigley, C. F., \& Herro, D. (2019). An educator's guide to STEAM. Engaging students using real-world problems (Juan Luis Fuentes). Prince, T. (2019). Ejercicios de mindfulness en el aula. 100 ideas prácticas [Mindfulness exercises for the class. 100 practical ideas] (José V. Merino Fernández).

145

\section{Call for papers}

Solicitud de originales

This is the English version of the research articles and book reviews published originally in the Spanish printed version of issue 275 of the revista española de pedagogía. The full Spanish version of this issue can also be found on the journal's website http://revistadepedagogia.org.

ISSN: 0034-9461 (Print), 2174-0909 (Online)

https://revistadepedagogia.org/

Depósito legal: M. 6.020 - 1958

INDUSTRIA GRÁFICA ANZOS, S.L. Fuenlabrada - Madrid 